\title{
The Investigation of the Properties of Thin Films by Means of X-rays. ${ }^{1}$
}

\author{
By Sir William BragG, K.B.E., F.R.S.
}

\begin{abstract}
THERE are a number of problems of the highest importance which are, or can be, contained in the study of what we often call the " thin film." They are linked together by the fact that most reactions between bodies are largely determined by what takes place at their points of contact, and therefore by the nature of their surfaces. What is to be found in the interior of the body is often of much less importance than the composition and state of its surface film. The immensely varied problems of surface tension are examples of one kind: the phenomena of catalysis, of friction and lubrication are examples of other kinds.
\end{abstract}

Though the thickness of the surface film is so important, it is often very small-beyond the limits of direct optical measurement. The X-rays measure more minute quantities than the microscope, and we may well ask if they can carry us any further. They measure only, it is true, the spacing of a stratification, and a stratification cannot consist of a single layer or film : so that the rays cannot be applied directly to the examination, for example; of the black spot on a soap film. But they are able to help in an indirect yet effective way. For they deal with problems of the arrangement of molecules, and all these manifestations of surface action are directly dependent on the arrangement of the surface atoms and molecules. Also the substances, the actions of which in single thin films are of great interest, are often found multiplied into crystals which actually can be examined by the X-rays.

The soap bubble and soap film have long been studied for their beauty and their interest. Since they have so large a surface in comparison with their volume content, they offer special advantages for the examination of surface actions. Yet they are so full of detail that even with the last few years new and most interesting discoveries have been made with respect to them. It will be convenient to review some of these briefly as illustrations of the facts that have to be accounted for.

When the bubble is near its end a black patch often appears, and soon afterwards the bubble bursts. The patch is black because it is so thin, and therefore reflects little light. It might be thought that the " black spot" represents a breakdown in the structure, a forerunner of collapse. But it is in reality a relatively stable affair : in proper circumstances it can be maintained for hours and days, as Sir James Dewar loved to show. Reinold and Rucker, Rayleigh, Johannot, and many others were greatly interested in the sharpness of its outline, its thinness, and the uniformity of its texture. They recognised two degrees of blackness, as Newton had done long before; they supposed them to represent a single film and its duplication. They succeeded in measuring the thickness, and estimated it to be about 60 Angström units in the case of the thinner and twice as much in that of the thicker.

When a film is correctly prepared and mounted, the black spot appears at once at the top of the film, a horizontal line separating it sharply from the rest with its horizontal bands of colour. Small black spots are continually forming at various points of the coloured film and rushing up to join the main spot: it is, of course,

Discourse delivered at the Royal Institution on Friday, January 16. the downward movement of their surroundings which makes them move in the opposite direction. Sometimes minute points of light like stars appear, moving about on the surface and especially at the edges of the black spots-drops of water apparently.

When observations of this kind are made in the quiet of a laboratory, with small and more manageable films and with special instrumental facilities, a fineness of detail is revealed which cannot be followed on the screen. Our knowledge of these details is due to the beautiful work of Perrin, published in 1918 , and to its repetition by Wells in I920. To state Perrin's results very briefly, it appears that the two degrees of thickness first observed are due to the existence of a very thin uniform film and its doubling, as had already been shown; that the existence of three more degrees of blackness observed by Johannot was due to further repetitions of the same layer, and that a close examination revealed the existence of dozens of these layers, all multiples of the same fundamental thickness. In the blackest spot there was but one layer, and this was, strangely enough, the most stable of all. Other similar layers could be found superadded, like sheets of paper of the same uniform thickness, until the film was thick enough to show the rich colours of Newton's rings which the soap film ordinarily displays. As we know, the colour of the film is an indication of its thickness. For example, the colours proceed from black through greys, becoming lighter and lighter as the thickness increases, then to a nearly pure white, then through straw-yellow, yellow, orange-red, dark red to a violet, which always changes very quickly with alteration of thickness, and so forms a definite stage. The thickness has now reached (for water) about 2 roo $\AA . U$. Perrin counted 37 to $3^{8}$ steps by which the superimposed sheets mounted to the full thickness that gave the violet tint. He made measurements also with monochromatic light and as his final result arrived at the conclusion that the thickness of the single film was about $5^{2} \AA$.U. Wells found a somewhat smaller value, namely, 42 A.U. Perrin, and Wells after him, concluded, on evidence which we have not time to consider, that the single film was composed of a double layer of oleic acid, arising from the hydrolysis of the sodium or potassium oleate in solution.

Now Rayleigh, Devaux, and more lately Langmuir, Hardy, and Adam have measured the thickness of an oil film spread on the surface of water. It appears that in the case of a fatty acid such as stearic or palmitic, when the surface is fully charged, the long chains stand on end, their carboxyl terminals rooted in the water, for which they have a great attraction, and their methyl terminals are turned outwards. The length of the oleic acid molecule is about $23 \AA$.U. It is clear that this length can easily be in agreement with the suggestion made by Perrin. His film of $5^{2}$ A.U., or $4^{2} \AA$ A.U. according to Wells, would consist of two layers of oleic acid molecules, the carboxyl ends meeting in the centre. The molecules are held together, side by side, to form a strong sheet, while the methyl groups form a surface reacting very slightly with anything outside. 
The examination of crystal forms by means of $\mathrm{X}$-rays shows that the peculiar arrangement of the molecules in these films on water extends also to the solid crystal in a great number of cases, and may probably be considered as characteristic of the structure of a large and important class of substances. It is in the first place to be found in the solid forms of the fatty acids, hydrocarbons, alcohols, and other long-chain molecules. These have been examined by Piper at Bristol, and by Muller and Shearer in the Davy Faraday Laboratory. I referred to a few of these a year ago. The number examined has been largely increased during the last iwelve months, and the results that have been obtained are ready for publication.

When a small quantity of one of these substances is placed upon a plate of glass or mica, either by melting or by pressing, a formation of layers results, much more so by the latter operation, for it would appear that the pressing and working encourage the arrangement of the molecules and the regularity of the layers that are formed. The material is now placed on an X-ray spectrometer, and a photograph is taken by the method of the revolving crystal. The photographic plate shows usually a number of lines which clearly represent the orders obtained by reflection from the plane of the layers. In this way the thickness of the layer can easily be measured with an accuracy of about I per cent. Ten or more orders are often observed. The cleavage plane of many crystals, especially of the class I am describing, often gives several orders, but not usually so many as in this case : other reflecting planes may give only one, perhaps two, higher orders of moderate strength, more often only very weak reflections, except in the first order. No doubt the case is parallel to the well-known effect in optics, where a grating yields many orders of spectra when the lines are sharp and fine. The "line " in this case is a welldefined discontinuity in the distribution of scattering centres which occurs at the ends of the long molecules; a defect would be caused by the presence of the hydrogens of a methyl group, an excess by the oxygens of a carboxyl group. We may suppose the planes which separate layers of these molecules to be well marked in this way, and that otherwise there is a fairly even distribution along the body of the molecule.

If we plot the spacing of each substance of any one series against the number of carbon atoms in the chain, we find at once that the indicating points lie exactly on a straight line, except when the chain is short. This has been proved by Muller and Shearer for the fatty acids, hydrocarbons, alcohols, ketones, and in other cases, and the regularity of the results leaves no doubt as to facts. The increase for each carbon atom is either about $\mathrm{x} \cdot \circ \AA$.U. or $\mathrm{I} \cdot 3 \AA$.U., the former occurring in the fatty acids, for example, and the latter in their esters.

If we may assume that the molecules lie perpendicularly to the layer, the numbers found for the thicknesses are actually the lengths of the molecules, and there is some ground for supposing this to be often true. But, of course, the molecules might not be normal to the layer, in which case the length of the molecule would be more than the thickness of the layer. It is certain that this may sometimes be so. Small crystals of substances of this class have been obtained and success- fully measured in these laboratories by R. E. Gibbs, in spite of the fact that they each weighed only about a hundredth of a milligram. Gibbs finds that the crystals are of monoclinic prismatic form.

In this instance, then, the molecule is not upright but sloping. It is difficult to imagine that the molecules all slope one way in the oil films on water; there would surely be some unique and recognisable direction in the film. It is possible that the explanation may be found in the fact that there are actually two ways, perhaps several ways, in which the molecules arrange themselves. Gibbs has found an orthorhombic as well as a monoclinic form; and Muller has observed that a hydrocarbon gives a somewhat different value for the spacing when it is only a few degrees below its meltingpoint. The physical appearance is different in the two cases. In the latter the film is translucent: in the usual case it is opaque, probably on account of minute crevasses formed during shrinking. This may be the cause of the change we see passing over cooling candle grease: it is transparent one moment, and suddenly clouds over.

The inclination of the molecule to the plane of cleavage must be finally settled before we are quite sure that we are measuring the actual lengths of the molecules, but we may hope for an early solution.

The fatty acid molecules are in double layers, but the hydrocarbons are not. We know this in two ways. In the first place, the actual increase in length for each carbon atom is twice as great in one case as in the other. In the second place, the even orders of the fatty acid spectra are very weak compared to the odd orders.

Such an effect can be produced in an optical grating by an alternation of white and black lines on a grey ground. An alternation of strong and weak black lines gives strength to the even orders: substituting white for one of the blacks is equivalent to changing the sign of its contribution. Gratings can be so made as to illustrate the point. If molecules pointing opposite ways are joined by their carboxyl terminations, then the methyl ends of the molecules are weaker in scattering centres than the general average along the molecule, but the parts where the carboxyl groups join together are above the average in strength.

Shearer finds another illustration of this effect in the case of hydrocarbons which form a single layer, but are converted into ketones by the substitution of oxygen for hydrogen at some point of the length. When the substitution is at the middle of the chain, there is at that point an excessive number of scattering centres, and we have again the circumstances that cause reinforcement of the odd orders. But if the substitution is not at the middle of the chain, the odd orders are no longer strong compared to the even.

We have, therefore, in these stratified layers which we are examining by the X-rays, the very same formation that Perrin has observed in the liquid films. The molecules are found, without exception, to be extended to their full length, and linked together by their carboxyl terminals. Shearer finds the length of the double molecule of oleic acid to be about 36 , which is to be compared with the 52 of Perrin and the 42 of Wells.

The conditions which lead to the formation of films on a water surface, and layers in the fatty acids and similar bodies, are operative also in the case of a large

NO. 2886 , VOL. I I 5$]$ 
number of more solid crystals. A certain flakiness is the result, the crystals cleaving very easily into thin layers which slide readily on one another, and often give a greasy feeling to the crystal.

Naphthalene and anthracene are cases in point. Their molecules are long and narrow, and are arranged side by side like the oleic acid molecules on water. A single layer is like the corn in a field; but they lean over like the corn when a wind is blowing. They are not perpendicular to the layer. The bonds that tie the molecules side to side must be stronger than those that tie them end to end, because the flakes are so easily parted from one another. The naphthalene molecule contains two hexagonal rings of carbon atoms and the anthracene molecule is extended by the addition of a third, so that the layer is thicker in the latter case. In both cases the molecule has a centre of symmetry, and in each layer the molecules are divided into two classes in respect to their orientation. Any member of one class is joined up by ties of some sort to several (probably four) members of the other class, which immediately surround it in the flake; and it would seem that this cross linking holds the flake together and gives it its strength.

It is likely that a very large number of other substances are built on the same plan. Even when their examination has not yet been attempted by means of $\mathrm{X}$-rays, their crystallographic measurements suggest the fact.

In other substances a similar external form is attained by a somewhat more complicated internal arrangement. The molecule has of itself no centre of symmetry, in fact no symmetry at all, and twice as many are required in the construction of the monoclinic prismatic unit of pattern. This is the case, for example, with benzoic acid, and probably with many of its derivatives. But there is the same flakiness which may be put down to the same causes : (I) the general orientation of the molecules so as to lie across the flake, and (2) the hydrogen terminations. In all these cases we find that the crystal is tied together by links extended from each molecule to neighbours of the other possible orientations: this seems to be an essential feature of crystal construction. It cannot, of course, be followed in the lowest type of crystalline symmetry, where there is but one orientation possible, and every molecule is arranged exactly as every other. But though this arrangement can be conceived, it is doubtful whether there is a known case: it has been shown by Astbury that calcium thiosulphate, generally quoted as the solitary example, has probably the symmetry of Class 2, which contains two orientations, connected by a centre of symmetry. Whether this is so or not, it is certain that in at least the vast majority of cases, cross linking is an important feature. It may very likely be a factor in the determination of the faces that appear on a crystal. A face will naturally contain specimens of more than one molecular orientation, so that the elements of the face may be tied together strongly. For example, in naphthalene, as in many other cases, all the usual faces contain equal numbers of molecules of the two orientations: one might even predict the arrangement from the knowledge of the form.

If, therefore, we survey the general characteristics of this large class of flaky crystals, we observe that the monomolecular films of Langmuir and the multiple stratifications of Perrin, and the whole range of "thin films," have much in common with the solid members of the class. Yet there are significant differences. It seems possible by studying both resemblances and differences to obtain some fresh light on the properties of the more " liquid " films.

With these facts before us we may perhaps formulate a. more detailed theory of the black spot on the soap film. The ordinary thick film is bounded on each side by the monomolecular film of oleic acid. In this film there is certainly arrangement. Adam, in particular, has examined the compression of the film under applied forces, and his observations and conclusions fall in naturally with the ordered array that we should expect to be there. But the film is imperfectly crystalline. It is compressible up to a certain point, and during the range of compression may be considered as a twodimensional gas. The perfect crystal of this class of substance is found in the films studied by Muller and Shearer, and in the minute crystal which Gibbs has measured. The perfect crystal of oleic acid contains twice as many orientations as the film of oleic acid on water: the molecules on the water are only one way up, and the reversed molecules are required to complete the structure.

Suppose, however, that the oleic acid films on the two sides come into contact anywhere; the conditions for the completion of the crystalline structure are now all present. The carboxyl groups not only meet, but interlace: each molecule in the upper group linking together two or four molecules in the lower, and vice versa. This is doubtless a far more stable form of arrangement than that of the single film. The two outside single films, once united in one place, must increase their area of contact, and will drive the expelled water before them until the accumulating heap becomes too great for them to push any further, the action being assisted by the tension of the rest of the film.

The film thus formed is a real crystal, because it contains all the molecular orientations. The black spot is simply the thinnest possible flake of oleic acid. It is true that oleic acid melts at $\mathrm{II}^{\circ} \mathrm{C}$., but the crystalline structure is there, in a mobile state. Solution cannot be made to enter between the two surfaces now united in crystalline fashion. Other films may be formed and float about on it, holding on by virtue of the feeble attractions of the methyl groups on one another : even the pressure of the air must help in keeping them together. These added films will slide about easily; they also will be true crystals of oleic acid, probably without any water.

The differences between the conditions and structure of the black spot on one hand and the neighbouring thick film on the other are so great that we may cease to wonder at the sharpness of the boundary and the enormous change in thickness: on one side of the boundary the thickness may be hundreds of times as great as on the other.

When the black spots appear and rise through the thick part of the film to join the general black area at the top, they leave trails behind them: they look like tadpoles swimming up to the top of the water. When the motion becomes less violent the tails shrink into small circular spots. On the other hand, if a thick 
patch is forced by the general turmoil into the middle of a black film, it tends also to a circular shape.

We come now to another of the important surface effects, namely, that of friction, or, stated inversely, of slipperiness. These flaky substances are in general slippery and greasy to the touch. The greasy feeling seems to be due to the ease with which the flakes are split from the main body of the substance, and then slide over it. Graphite is an extreme example of the flaky state: and without forcing its inclusion in the class of substances we are considering, we observe that the atoms in each flake are tightly tied together, and that there is a very weak linkage between a flake and its neighbours. It is the combination of these conditions that makes for good lubricating qualities. Now in these substances the same conditions hold to a greater or less extent: the molecules of stearic acid, for example, are tied together more tightly side to side than across the ends at which the methyl groups are attached. If, therefore, stratification exists to any considerable degree, the same consequences follow as in the case of graphite. In general, stratification is incomplete, which is another way of saying that a large perfect crystal of stearic acid is never seen. Pressure is one agent that causes stratification, so that if one presses a piece of stearic acid or other material, the very pressure produces the conditions for easy slipping. I have already mentioned that Shearer and Muller have often found that a portion of the material melted on to a piece of mica or glass, and placed on the spectrometer in the necessary position for giving reflections from planes parallel to the glass, was comparatively ineffective in this respect. It would give clear evidence of those two spacings which are found on all the plates, and are ascribed to the widths of the molecules and are independent of their lengths. When the specimen was pressed, or rubbed down on to the plate, the stratification spacing appeared at once, and the others disappeared, thus showing the nature of the rearrangement that had taken place. It may be that this effect explains other properties of greases. I am told that the special grease used in binocular fittings is made serviceable by working with a palette knife.

We must recognise, however, that the layers built into the crystal are not at all times ready to slide, otherwise a mere tilt of the crystal would cause them all to slide off one another like a pack of cards. There is a sticking friction to be overcome, exactly as in graphite. It is only possible to speculate as to the cause : perhaps it is a real molecular effect; and sliding only occurs when the bonds, weak as they are, are further weakened, as if the substance was about to melt: perhaps it is rather a mass effect and due to imperfect crystallisation.

It seems not unlikely that, at its best, slipperiness in these cases is almost perfect. Between two perfectly formed methyl layers there is very little friction indeed, perhaps none at all. The methyl layer is seen at its best on the surface of the black spot in the soap film, and various writers have noted that one layer slides very easily over the other. Perrin, in fact, has directed attention to this remarkable effect, as shown in the Brownian movement of fragments of one layer, lying on and sliding over another.

In some of the experiments of Sir William Hardy and Miss Doubleday the condition of nearly complete slipperiness is attained. Their beautiful researches on " boundary lubrication," that is to say, the slipperiness of very thin films, have established certain rules of surprising simplicity. They have found cases in which friction nearly vanishes, and in general accordance with the above, they frequently occur when a solid lubricant is practically wiped and rubbed off the surface, a true stratification in a very thin film being probably left.

Not only the phenomena of surface tension and of lubrication, but also those of catalysis, must be intimately connected with the actual arrangement of molecules. We speak of stereochemistry as showing the relations of the atoms in the molecules to one another, their mutual orientation and distances. To deal with these subjects we shall have to extend stereochemistry to cover the mutual distances and orientations of molecules as well as of atoms. We see that we must not treat a molecule as if it were simply a sphere attracting according to gravitational laws: it is not even sufficient to speak of an atom in this way, except as an approximation in the case of ionic substances like rock-salt. If some super-Brobdingnagian inquirer were to argue from effects observed on the surface of the earth as to the nature of the human beings to which the effects were ascribed, and being unable to detect a single individual by the most refined methods of which he was capable, should say, "Let us provisionally assume the human being to be a sphere, having similar properties in all directions and no special points of attraction," he would not get very far towards a satisfactory explanation of his subject. It would be an advance should he recognise the existence of two types of opposite sign, and lay the foundations of an ionic theory of heteropolar assemblages, but even then he would fall far short of the truth. In the same way, when we try to explain surface tension as the result of the mutual gravitational attraction of spherical atoms or molecules, we cannot make much progress. Indeed, we sometimes arrive at consequences that appear startling, as when we determine their attractions by measuring the energy required to tear surface molecules away in the process of evaporation, and then proceed to deduce the existence of pressures of thousands of atmospheres within the body of a liquid. So we may measure the force required to tear away the fringing links of a piece of chain mail, take them to be due to mutual attraction between the links, and thence deduce the existence of an enormous pressure within the piece.

It is when we consider a catalytic surface as possessing active centres on its surface, the relative positions, magnitudes, and mutual distances of which are such that two wandering molecules of different kind, attracted by these points, may be held together in a special way, that we get some idea of the fundamental action of catalysis. It is important to consider, as the basis of surface actions, the arrangements of the molecules at a surface, both arrangements that actually exist, and those that are predisposed to exist. This idea runs through all the work of those experimenters, Langmuir, Harkins, Hardy, Adam, Perrin, and many others who have made such progress of recent years: the X-ray studies emphasise this view and supply many quantitative measurements by which it may be shaped and strengthened.

$$
\text { NO. } 2886 \text {, VOL. I I } 5]
$$

